\title{
ARTICLE
}

\section{Recent advances of Targeted Radioisotope Therapy (TRT) research (PLENARY)}

\author{
Tatsuya Higashi* \\ Department of Molecular Imaging and Theranostics, National Institute of Radiological Sciences (NIRS), National Institutes for \\ Quantum and Radiological Science and Technology, Anagawa, Chiba, 263-8555, Japan
}

\begin{abstract}
QST and our department were established in April 2016, focusing the researches in targeted radioisotope therapy (TRT). Recently, TRT attracted attention because a newly-developed commercially available pharmaceutical, Ra-223 chloride (trade name; Xofigo), an alpha particle emitting TRT agent. Despite the conventional TRT agents, such as I-131, Sr-89 and Y-90, are beta emitting phamaceuticals, Ra-223 chloride is a first-ever alpha particle emitting TRT product and shows a strong therapeutic effect in patients with bone metastases from prostate cancer because of its poweful cell killing effect of alpha particle irradiation. In TRT research fields, there are several other promising alpha particle emitting TRT agents, and one of these promising alpha particle emitting TRT agents is Ac-225 labelled PSMA-617 (Prostate Specific Membrane Antigen-617), which is a newly developed TRT agent and showed a suprising therapeutic effect in two metastatic prostate cancer patients in end-stage (case report in J Nucl Med 2016). QST and our department recently developed and reported one promising candidate for TRT, meta- At-211 astato-benzylguanidine (At-211-MABG). At-211-MABG is also an alpha-emitting radiopharmaceutical targeting the treatment of neuroendocrine tumors, such as malignant pheochromocytoma. In this session, we would like to show our recent advance in the research of At-211-MABG. One of our research goals is to develop Japan's first-ever Japanese-made pharmaceutical products for TRT including Ac-225 labelled TRT agents. In addition, we would like to show our strategies in the future development of preclinical and clinical researches, dosimetric researches, and regulatory science, especially in the field of alpha emitter TRT pharmaceutical products.
\end{abstract}

Keywords: Ra-223 chloride, Ac-225 labelled PSMA-617, At-211-MABG

\section{Introduction}

What is the most expensive medical drug available in Japanese Health Insurance? That is radiolabeled (yttrium-90) anti-CD20 monoclonal antibody: Ibritumomab Tiuxetan. Its medical fee in Japan is about 40,000 dollars. This therapy is one of the typical type of Targeted Radioisotope Therapy (TRT). In TRT, cytotoxic isotopes accumulate to target molecules of cancer cells by ligand (small molecules or hormones) or antibodies, and then cancer cells are destroyed by radiation from isotopes. An effective TRT can be accomplished with an appropriate combination of target molecules, ligands or antibodies, and isotopes (Figure 1). However, there have been several problems in the development of TRT, such as: 1) There have been only rare diseases in case of target tumors until recently, 2) Difficulties in labelling methods of ligands and antibodies with the isotopes, 3) Only beta emitter isotopes are largely available till now, and 4) In case of iodine-131 (gamma and beta emitter), its clinical treatment needs radioisotope (RI) wards because of the risk of radiation exposure to other persons (medical staffs and general people). Recently, new technologies in cyclotron and labelling were brought to clinical practice and newly-developed TRT, especially Targeted Alpha Therapy (TAT) is becoming increasingly popular every year.

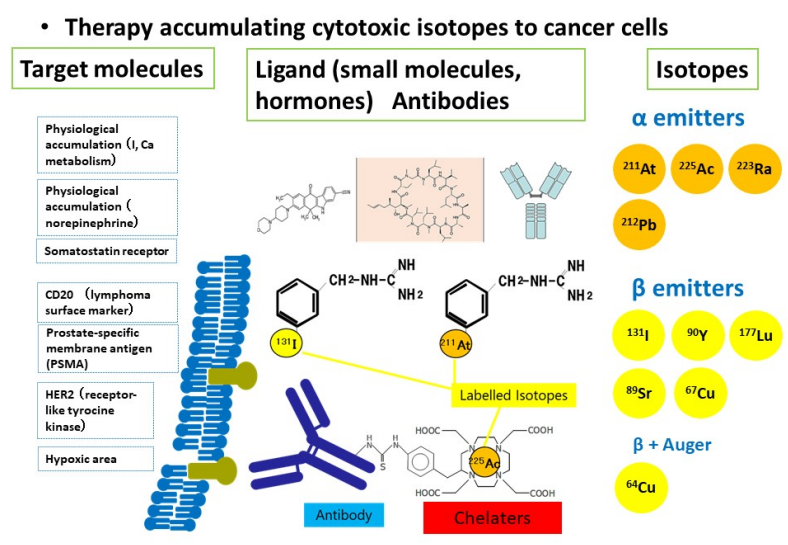

Figure 1. Targeted radioisotope therapy/TRT.

*Corresponding author. Email: higashi.tatsuya@qst.go.jp 


\section{Principles of TRT}

Beta emitters, such as yttorium-90 (Y-90), have relatively longer tissue penetration (millimeter level). Therefore, side effect due to the relatively high radiation exposure to surrounding normal tissues is one of the major problems in case of Y-90, especially in bone marrow. Because of this side effect, TRTs using beta emitters are always limited in its maximum administered dose, which results in ineffective treatment outcome. In addition, TRT using iodine 131, a typical beta emitter with gamma ray, needs specially-equipped treatment rooms or wards for TRT, to minimize the radiation exposure of gamma ray to medical staff and general people. Besides these, the construction and annual maintenance of TRT rooms are very expensive. In Japan, the shortage of TRT rooms is one of the problems in society and medical fields $[1,2]$.

In contrast, several alpha emitters have been brought to medical field because of the innovation of technology. Alpha emitters are known to have high LET (linear Energy Transfer) and high RBE (Relative Biological Effectiveness), which results in high anti-cancer effect with double-strand breaks of cancer DNA independent from the presence of hypoxia in tumor tissues. Alpha emitters have short tissue penetration (micrometer level), which is comparable in length to 1-2 cancer cells. As a result, normal tissues can be maintained radiation-exposure-free even if a normal organ is located next to a cancer nodule. Radiation protection can be also easily guaranteed by paper and aluminum plate. Hence, there is no need to have specially-equipped treatment rooms or wards for TRT. All these characters of alpha emitters indicate that TRT using alpha emitters is ideal for cancer treatment with radiation safety and high effectiveness.

\section{Radium-223 dichloride}

Radium-223 dichloride, Xofigo, is the very first TRT agent using alpha emitter in the world. From 2016, it is commercially available in Japan with health insurance coverage. Its supply is attained by a generator system of actinium-227 (Ac-227). Its manufacturing process is followed by the elution and purification of Ac-227, and the final agent, radium-223 dichloride, is produced after the formulation and final sterilization.

Alpha therapy with radium-223 dichloride is used for the treatment of patients with castration-resistant prostate cancer (CRPC), symptomatic bone metastases without known visceral metastatic disease. Prostate cancer is known to metastasize to the bone most often, and bone metastasis is a major cause of mortality and morbidity in CRPC. Current bone agents in metastatic prostate cancer are palliative. Short comings of current therapies are detrimental side effects and the lack of overall survival benefit. Phase III clinical trial of Xofigo was performed in an international framework and its result was published in the New England Journal in 2013 [3], which showed the risk of death among patients in the radium-223 group was reduced as compared with the placebo group. On an out-patient basis, in the practical clinical situation, Xofigo can be administered intravenously maximum 6 times for one patient with four weeks interval. There is no need to have specially equipped TRT rooms and side effects are also known to be minimal.

Clinical market size of Xofigo is now glowing up to hundreds of millions of dollars in USA (2014). In Japan, after one year from the approval of insurance, the number of patients treated by Xofigo is about one thousand (2017).

\section{TRT of Prostate-specific membrane antigen (PSMA)}

Prostate-specific membrane antigen (PSMA) is the type II membrane glycoprotein with 750 amino acids. It is known to express in normal membrane of prostate, membrane of prostate cancer cells, and membrane of other organ and cancers. High expression of PSMA is also known be observed in prostate cancers with castration-resistant prostate cancer (CRPC) [4]. Therefore, PSMA is one of the appropriate targets for TRT of prostate cancer. PSMA-targeted TRT for CRPC with bone metastasis has been performed by lutetium-177 (Lu-177) [5], a beta emitter with a maximum energy of $0.5 \mathrm{MeV}$ and a maximal tissue penetration of $0.23 \mathrm{~mm}$ for beta ray $(78.6 \%)$. Lu-177 is known to have a physical half-life of 6.64 days and emits low energy gamma rays at $208 \mathrm{KeV}$ with $11 \%$ abundance. It already showed a useful therapeutic results in USA and European countries and a phase II clinical trial by a pharmaceutical company is now going on in USA $\left({ }^{177}\right.$ Lu PSMA-617).

Recently, a report of two cases treated by PSMA-targeted TRT for CRPC with bone metastasis using alpha emitter, actinium-225 (Ac-225), was published in 2016 in the Journal of Nuclear Medicine [6]. In this paper, two cases of CRPC patients in end stage were treated by TRT using ${ }^{225}$ Ac PSMA. Both of these cases showed complete emission (CR) with serum PSA (tumor marker of prostate cancer)-free after the three or four cycles of TRT. This case report is an epoch-making paper of TRT not only for nuclear medicine physicians but also for general oncologists because of its surprising therapeutic effect without major side effects. So far, TRTs using beta emitters have been supposed to have limited therapeutic effect with a certain side effect of bone marrow and other organs. However, this TRT using ${ }^{225}$ Ac PSMA accomplished CR in two end-stage cancer patients without major side effect. Recently, only limited information of further studies with more patients is disclosed, in which therapeutic effects are supposed to be superior to other TRT using beta emitters. Concerning the side effects, there is no major side effects while the harmful event is only limited in salivary glands [7].

Actinium-225 is known to have 10 days of physical 
half-life and its tissue penetration is 47.1 um for alpha ray, with a large cumulative energy of $28 \mathrm{MeV}$. So far, the production of Ac-225 is only performed by tholium-229 generator. Therefore, at present, the production of Ac-225 is the main technological research target in the world.

\section{Conclusion}

For some decades, TRT with beta emitters has been only available in limited cancers and medical condition in Japan has disrupted the growth of TRT. Recent appearance of TRT using alpha emitters has changed this situation with its high therapeutic effect and its radiation safety features from excess radiation exposure. Several more Alpha emitters or Actinides with short half-life are the targets of research and clinical use for TRT in Medical Fields. For further development of TRT, we still have several problems to solve in order to apply TRT in the clinical practice (Table 1). The clinical use of TRT with alpha emitters is still limited so far, but its development is widely expected.

Table 1. Targeted Radioisotope Therapy (TRT) and Targeted Alpha Radioisotope Therapy (TAT): Problems for further development.

- Target tumor: common and therapy-reflactory disease (pancreatic ca, etc) would be a main target

- Ligand or Antibody: Further development of labelling methods with alpha emitters

- Isotope: Provide enough amount of raw materials (reactor fuels, international trade law, etc)

- Isotope: Establish sophisticated methods for alpha emitters production

- Isotope: Establish management of radioactive wastes

\section{References}

[1] T. Higashi, R. Nishii, S. Yamada, et al., Delayed initial radioactive iodine therapy resulted in poor survival in patients with metastatic differentiated thyroid carcinoma: a retrospective statistical analysis of 198 cases, J. Nucl. Med. 52 (2011), pp. 683-689.

[2] T. Higashi, T. Kudo and S. Kinuya, Radioactive iodine (131I) therapy for differentiated thyroid cancer in Japan: current issues with historical review and future perspective, Ann. Nucl. Med. 26 (2012), pp. 99-112.

[3] C. Parker, S. Nilsson, D. Heinrich, et al., The homodimer of prostate-specific membrane antigen is a functional target for cancer therapy, Proc. Natl. Acad. Sci. USA, 22 (2003), pp. 213-223.

[4] N. Schulke, O.A. Varlamova, G.P. Donovan, et al., The homodimer of prostate-specific membrane antigen is a functional target for cancer therapy. Proc. Natl. Acad. Sci. USA, 28 (2003), pp. 12590-12595.

[5] K. Rahbar, H. Ahmadzadehfar, C. Kratochwil et al., German multicenter study investigating ${ }^{177}$ Lu-PSMA-617 radioligand therapy in advanced prostate cancer patients, J. Nucl. Med. 58 (2017), pp. 85-90.

[6] C. Kratochwil, F. Bruchertseifer, F.L. Giesel, et al. ${ }^{225}$ Ac-PSMA-617 for PSMA-targeted $\alpha$-radiation therapy of metastatic castration-resistant prostate cancer, J. Nucl. Med. 57 (2016), pp. 1941-1944.

[7] C. Kratochwil, F. Bruchertseifer, H. Rathke, et al., Targeted $\alpha$-therapy of metastatic castration-resistant prostate cancer with ${ }^{225}$ Ac-PSMA-617: dosimetry estimate and empiric dose finding., J. Nucl. Med. 58 (2017), pp. 1624-1631. 